\title{
Growth and Characterization Studies of MnHP Single Crystal in Silica Gel Medium
}

\author{
P. Suresh ${ }^{2}$, G. Kanchana ${ }^{1}$, P. Sundaramoorthi*2 \\ ${ }^{1}$ Department of Biochemistry, Muthayammal College of Arts and Science, Rasipuram, Namakkal, \\ India-637408. \\ ${ }^{2}$ Department of Physics, Thiruvalluvar Govt. Arts College, Rasipuram, Namakkal, India-637401. \\ *Corresponding Author, contact: moorthi.sundara@gmail.com \\ Phone: 04287231802, Fax: 04287231882
}

\begin{abstract}
MnHP (Manganese Hydrogen Phosphate) crystals were grown in silica gel medium using different gel densities, various concentrations of ortho- phosphoric acid and supernatant solutions in single diffusion process. The gel pH plays an important role in the formation of different $\mathrm{HPO}_{4}$ species in the phosphoric system. The $\mathrm{pH}$ range in which $\mathrm{HPO}_{4}{ }^{2-}$ ions dominates were considered which in turn is necessary for the growth of MnHP crystals. The characterizations of grown crystals were studied by FTIR, SEM, XRD and etching. The results are reported and discussed in detailed manner.
\end{abstract}

Key Words: MnHP, calculi, surface morphology, growth parameters, trace element and SMS gel medium.

\section{INTRODUCTION}

SHP (Strontium hydrogen phosphate) and BHP (Barium hydrogen phosphate) were grown in silica gel medium at room temperature and reported [1-2]. In the present investigation, single crystal of trace element was grown in silica gel medium at different parameters, which contains one major element (Phosphate), and one minor or trace element (Manganese). MnHP is a single crystal which typically represents the biological crystals formed in the human urinary tracts called renal stones. The body of a normal man weighing $70 \mathrm{~kg}$ contains about $12-20 \mathrm{mg}$ manganese. It is distributed throughout the body tissues and fluids. Bone, kidney, liver, pancreas and pituitary contain more manganese than other tissues. The manganese 
content of human blood is very low ranging from 2 to 3 micrograms per $100 \mathrm{ml}$. Only 3-4 per cent of the manganese present in the regular diet is absorbed and mixed in plasma and the remaining being excreted in faeces. A normal human body requirement of manganese ranges from about 2.5 to $5.0 \mathrm{mg}$ per day.

Manganese is present naturally in many foods such as spices, sea foods, cereals, grains and leafy vegetables. There are some evidences that manganese is essential for the growth of animals, especially for the normal skeletal growth in the prenatal period. Manganese appears to play an important role in the functioning of central nervous system. It is also essential for the normal reproductive function. If the mineral level of the body fluid increases, automatic mineral deposition starts leading to the development of renal stones. Authors have done a series of experiments with silica gel crystal growth medium at different $\mathrm{pH}$ values ranging from 5.5 to 11 . One can obtain the periodic precipitation, Liesegang rings [3-5] of biological crystals named as HAP, Brushite, Struvite, BMHP, SMHP etc.

\section{MATERIALS AND METHODS}

The dissociation of ortho-phosphoric acid system can be represented by three-dissociation equilibrium and the presence of various ions at various $\mathrm{pH}$ values are reported [6]. Based on these results, the gel $\mathrm{pH}$ in the range from 6 to 11 has been used (Milwaukee QS-MN pH-600, packet digital $\mathrm{pH}$-meter used for measurements) in which the $\mathrm{HPO}_{4}{ }^{2-}$ ion dominates or alone exists. This decreases the possibility of the occurrence of MnP crystals during MnHP growth. The crystallization apparatus employed were glass test tubes of $25 \mathrm{~mm}$ diameter and $150 \mathrm{~mm}$ long for single diffusion method (SDM). The chemicals used were Excelar-Qualigens(E-Q) AR grade $\mathrm{MnCl}_{2}$ and E-Q AR grade ortho-phosphoric acid (Sp.gr.1.75). The SMS gel or water glass was prepared as per the literature [7]. One of the reactant ortho-phosphoric acid was mixed with silica gel at desired gel density and elevated temperatures. After the gel set, the supernatant mixture (Manganese chloride) at a required mole solution was slowly added along the walls of the growth columns (test tubes) over the set gels and tightly closed to prevent evaporation. Then the growth systems were allowed to react within the gel medium and the following chemical reaction take place.

$\mathrm{MnCl}_{2}+\left[\mathrm{H}_{3} \mathrm{PO}_{4}+\right.$ gel $] \rightarrow \mathrm{MnHPO}_{4}+\mathrm{By}$ - products

\subsection{The following observations have been made in the present investigation}

The reaction starts immediately after the addition of supernatant solutions. But the nucleation was observed only after 26 hours and the growth process took a period of nearly one year for completion. Some well-developed single, poly crystals were observed in the SDM growth columns. The gel density above $1.06 \mathrm{gm} / \mathrm{cc}$ and with $\mathrm{pH}$ above 8 yielded no crystals, but mean time reaction takes place. Some of the test tubes of gel density $1.03 \mathrm{gm} / \mathrm{cc}$ with $\mathrm{pH}$ around 6.5 
and below 6 were allowed for the reactions to take place. After a period of five months, a less number of nucleation was observed near the bottom and middle of the test tubes. Some of them grew as a needle and well transparent single and platelet crystals. From these investigations, the optimum growth parameters of MnHP crystals were identified and reported in Table-1.

Table-1 MnHP crystal growth parameters in SDM

\begin{tabular}{|c|c|c|c|c|c|c|c|}
\hline $\begin{array}{l}\text { Gel } \\
\text { density } \\
\text { gm /cc }\end{array}$ & $\begin{array}{l}\text { Ortho- } \\
\text { phosphoric } \\
\text { acid } \\
\text { concentration } \\
\text { in N }\end{array}$ & $\begin{array}{l}\mathrm{Gel+} \\
\mathrm{H}_{3} \mathrm{PO}_{4} \\
\mathrm{pH} \\
\text { value }\end{array}$ & $\begin{array}{l}\text { Gel } \\
\text { setting } \\
\text { time in } \\
\text { hrs }\end{array}$ & $\begin{array}{l}\text { Supernatant } \\
\text { concentration } \\
\mathrm{MnCl}_{2}(\mathrm{M})\end{array}$ & $\begin{array}{l}\text { Nucleation } \\
\text { observed in } \\
\text { hrs }\end{array}$ & $\begin{array}{l}\text { Growth } \\
\text { period in } \\
\text { days }\end{array}$ & $\begin{array}{l}\text { Types of crystals } \\
\text { observed in all } \\
\text { conditions and } \\
\text { harvested crystal } \\
\text { size }\end{array}$ \\
\hline \multirow[b]{2}{*}{1.04} & 1 & $\begin{array}{l}6.4 \\
6.8 \\
6.9 \\
7.3\end{array}$ & $\begin{array}{l}26 \\
16 \\
1 \\
18\end{array}$ & $\begin{array}{l}2 \\
- \text { do- } \\
- \text { do- } \\
\text {-do- }\end{array}$ & $\begin{array}{l}26 \\
37 \\
42 \\
99\end{array}$ & 280 & \multirow{4}{*}{$\begin{array}{l}\text { Single, } \\
\text { Poly crystals, } \\
(2 \mathrm{mmx} \quad 2 \mathrm{~m}\end{array}$} \\
\hline & 1.5 & $\begin{array}{l}6.6 \\
6.9 \\
7.1 \\
8.0\end{array}$ & $\begin{array}{r}28 \\
1 \\
3 \\
46\end{array}$ & $\begin{array}{l}\text {-do- } \\
\text {-do- } \\
\text {-do- } \\
\text {-do- }\end{array}$ & $\begin{array}{l}16 \\
26 \\
56 \\
67\end{array}$ & 290 & \\
\hline \multirow[b]{2}{*}{1.05} & 1 & $\begin{array}{l}6.3 \\
6.8 \\
6.9 \\
7.4\end{array}$ & $\begin{array}{l}34 \\
6 \\
1 \\
68\end{array}$ & $\begin{array}{l}\text {-do- } \\
\text {-do- } \\
- \text { do- } \\
\text {-do- }\end{array}$ & $\begin{array}{l}22 \\
72 \\
48 \\
88\end{array}$ & 210 & \\
\hline & 2 & $\begin{array}{l}6.6 \\
6.9 \\
7.1 \\
7.5\end{array}$ & $\begin{array}{l}24 \\
1 \\
12 \\
48\end{array}$ & $\begin{array}{l}\text {-do- } \\
- \text { do- } \\
- \text { do- } \\
\text {-do- }\end{array}$ & $\begin{array}{l}43 \\
30 \\
44 \\
82\end{array}$ & 275 & \\
\hline
\end{tabular}

An extension of the reaction period even up to one and a half year did not improve the size of these crystals. The growth columns (SDM) of MnHP crystals and harvested MnHP crystal are shown in Fig-1 and Fig-2 respectively. The maximum dimension of the crystals obtained is 2 $\mathrm{mm} \times 2 \mathrm{~mm} \times 2 \mathrm{~mm}$. In this investigation fast gel set shows non-transparent gel medium and gives less nucleation and yield.

\section{CHARACTERIZATION STUDIES OF MnHP CRYSTALS}

\subsection{FTIR spectral analysis of MnHP crystal}

FTIR spectrometer having $\mathrm{KBr}$ pellets sample holder and $\mathrm{KBr}$ detector was used for the analysis. The KBr pellet samples were used and the absorption frequencies range from 600 to $4000 \mathrm{~cm}^{-1}$ [8-12]. The absorption bonds, absorption frequencies and percentage of transmittance were 
compared with the reported values. The values are tabulated in Table- 2 which confirms the presence of MnHP crystal constituents.

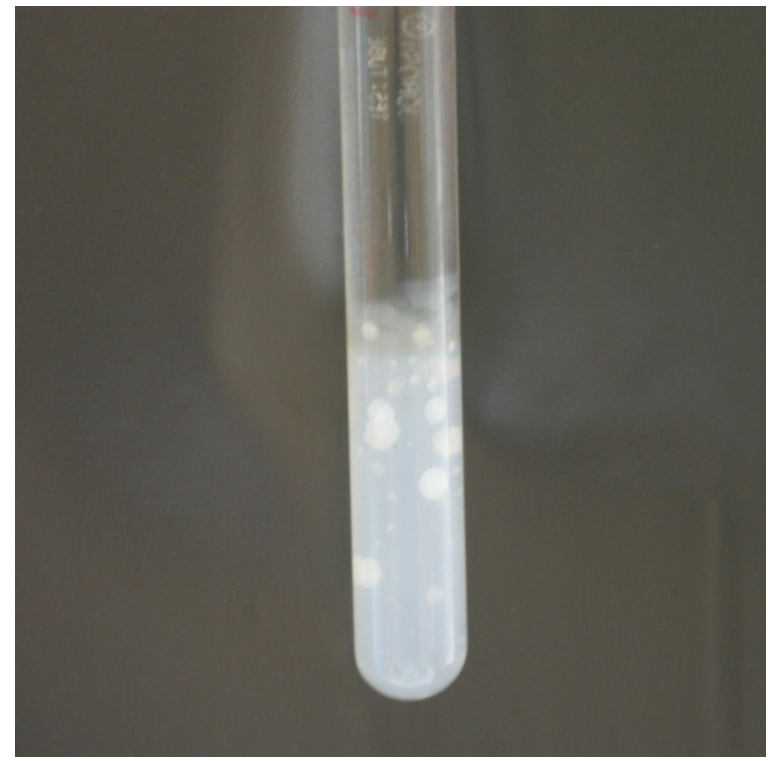

Fig-1

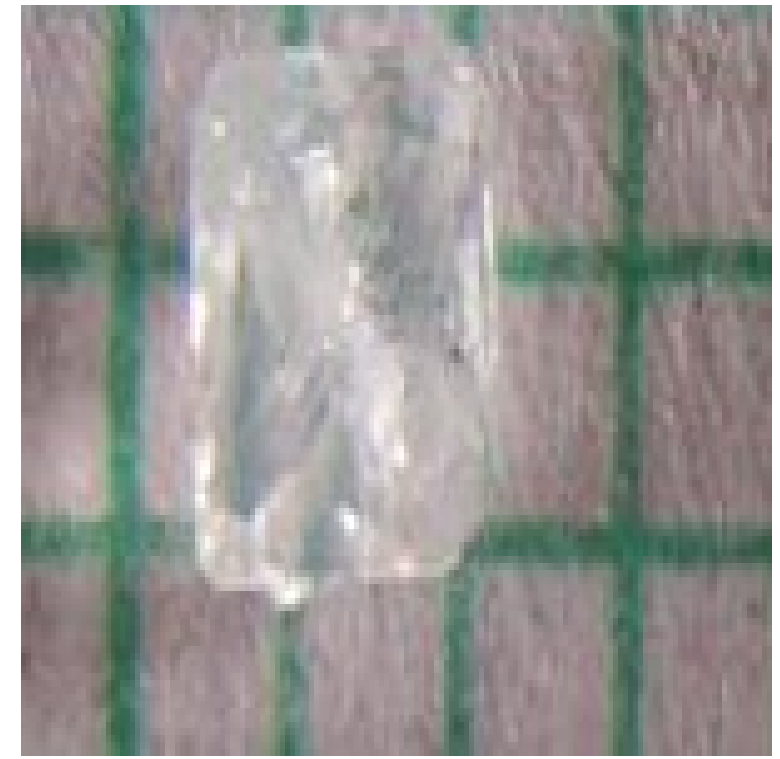

Fig-2

Fig-1 Growth of MnHP crystals within laboratory environment

Fig-2 Harvested MnHP well transparent crystal

Table-2 FTIR spectral analysis of MnHP Crystal

\begin{tabular}{|l|l|l|l|l|}
\hline S.No. & Composition/Bond & $\begin{array}{l}\text { Absorption } \\
\text { frequency } \\
\text { Reported } \\
\text { value } \\
\text { cm-1 }\end{array}$ & $\begin{array}{l}\text { Absorption } \\
\text { frequency } \\
\text { Observed } \\
\text { value cm-1 }\end{array}$ & $\begin{array}{l}\text { \% of } \\
\text { transmittance }\end{array}$ \\
\hline $\mathbf{1}$ & Manganese \& hydrogen & 3477 to 3207 & 2811 & 36 \\
& O-H symmetric, & & 3559 & 23 \\
& asymmetric (in plane) & & 3497 & 19 \\
\hline $\mathbf{2}$ & O-H out of plane & 745 & 3310 & 22 \\
\hline $\mathbf{3}$ & PO group & 1000 to 1100 & 1131 & 47 \\
& & & 1050 & 12 \\
& & & 989 & 36 \\
\hline $\mathbf{4}$ & Manganese/Apatite & $600-1010$ & 546 & 23 \\
& group & Frequency) & 1086 & 31 \\
& & & & 14 \\
\hline
\end{tabular}




\subsection{Etching study of MnHP crystal}

A well-grown MnHP crystal was immersed in $\mathrm{HCl}$ solution at a desired concentration. The dissolution of MnHP crystal depends upon the etchant concentration, temperature, crystal morphology, etching time etc. [13-16]. The etch pits are shown in Fig-3. The etch pits observed in the photo are cone pits, leaf pits and step pits.

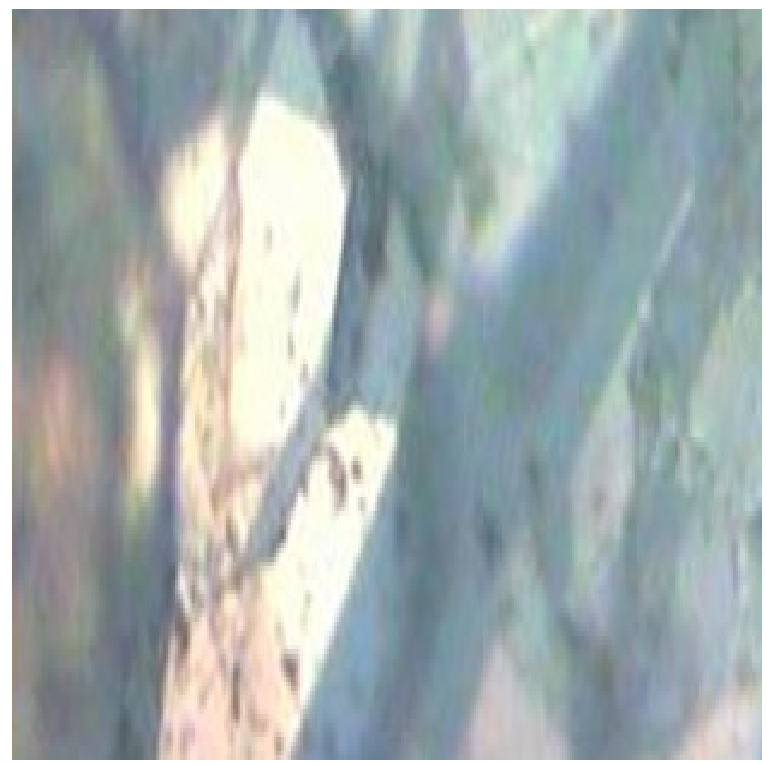

Fig-3 Etch photo of MnHP crystal at room temperature, HCL as an etchant, etching time - 7 minutes, etchant normality $-2 \mathrm{~N}$

\subsection{Scanning Electron Microscopic studies of MnHP crystal}

A well-grown MnHP single crystal was selected for the investigation of surface morphology by using SEM. The SEM photograph was made in the version S-300-I instrument. The sample named VCA-600 kept in lobe middle; the data size was $640 \times 480 \mu \mathrm{m}$. The minor and major magnifications of SEM were about 250 times. SEM acceleration voltage was 25000 volts and the sample was kept in a high vacuum. 18200 $\mu$ m working distance and monochromatic color mode was employed. $200 \mu \mathrm{m}$ focusing of MnHP crystal SEM is shown in Fig-4. In the surface analysis of SEM picture of MnHP crystal, smooth, fine grain boundaries and few valley regions are observed [17-20]. 


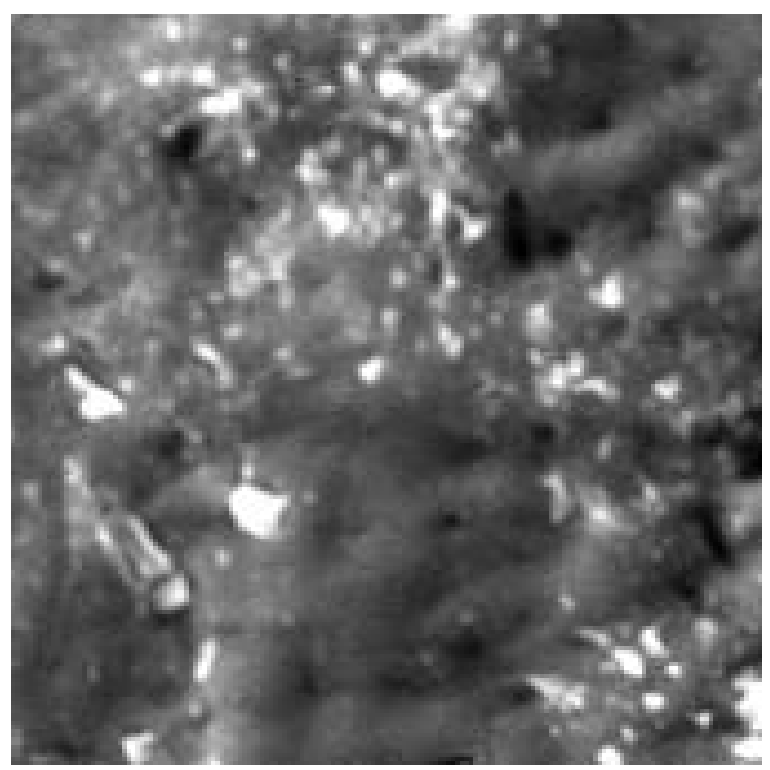

Fig-4 SEM picture of MnHP crystal

\subsection{X-ray diffraction of MnHP crystal}

The XRD results revealed the crystalline property of crystal. The XRD pattern and diffraction indices of the MnHP crystals were recorded. Using the programme (Proszki) the lattice parameters of the MnHP crystal were calculated.

CD0> lo

From to: 1- 25

$$
\begin{aligned}
& \text { 1H -3. 1. -4. * } 10.00 \mathrm{~S}-3.38 \quad 9.10 \quad 2.70 * * \mathrm{~S}-20.782636 .5 \\
& 2 \mathrm{H}-2 . \quad 1 . \quad-5 . * 10.59 \mathrm{~S} \quad 12.97 \quad 10.73 \quad-1.05 * * \mathrm{~S}-10.76 \quad 1819.2 \\
& 3 \mathrm{H}-4 . \quad 1 . \quad-3 . * 10.17 \mathrm{~S}-20.14 \quad 7.80 \quad 6.67 * * \mathrm{~S} \quad 10.70 \quad 412.8 \\
& 4 \mathrm{H}-4 . \quad 1 . \quad-4 . * 11.38 \mathrm{~S}-12.58 \quad 9.53 \quad 6.56 * * \mathrm{~S}-10.75 \quad 1394.4
\end{aligned}
$$

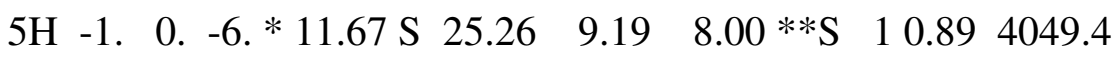

$$
\begin{aligned}
& 6 \mathrm{H} \quad 0 . \quad 0 . \quad-6 . * 11.47 \mathrm{~S} \quad 35.97 \quad 10.46 \quad 4.08 * * \mathrm{~S} \quad 20.813206 .0 \\
& 7 \mathrm{H}-2 . \quad 1 . \quad-6 . * 12.37 \mathrm{~S} \quad 16.63 \quad 12.09 \quad-0.23 * * \mathrm{~S} \quad 10.8111234 .1 \\
& 8 \mathrm{H}-4 . \quad 2 . \quad-4 . * 11.91 \mathrm{~S}-9.79 \quad 13.59 \quad-5.82 * * \mathrm{~S} \quad 10.8013123 .8 \\
& 9 \mathrm{H}-1 . \quad 1 . \quad-6 . * 11.85 \mathrm{~S} \quad 26.61 \quad 13.03 \quad-4.21 * * \mathrm{~S} \quad 10.891140 .9 \\
& 10 \mathrm{H}-4 . \quad-2 .-2 . * 9.86 \mathrm{~S}-43.00 \quad-8.36 \quad 54.71 * * \mathrm{~S} \quad 10.7217711 .2 \\
& 11 \mathrm{H}-1 . \quad-3 . \quad-4 . * 9.94 \mathrm{~S} \quad 17.37 \quad-9.01 \quad 56.58 * * \mathrm{~S} \quad 30.7428197 .4 \\
& 12 \mathrm{H}-4 . \quad-3 .-3 . * 11.67 \mathrm{~S}-32.09 \quad-9.59 \quad 62.50 * * \mathrm{~S}-20.641967 .7 \\
& 13 \mathrm{H}-3 . \quad-3 . \quad-4 . * 11.49 \mathrm{~S}-11.35 \quad-8.21 \quad 58.57 * * \mathrm{~S} \quad 10.67 \quad 582.6
\end{aligned}
$$

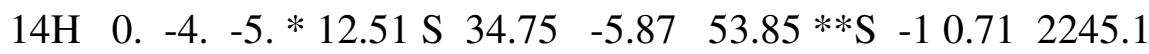

$$
\begin{aligned}
& 15 \mathrm{H}-4 . \quad-2 . \quad-4 \text {. } * 11.90 \mathrm{~S}-19.11 \quad-3.32 \quad 45.32 * * \mathrm{~S} \quad 20.6824786 .3
\end{aligned}
$$

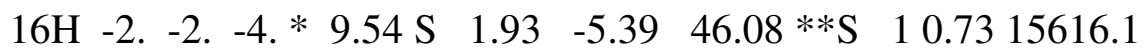




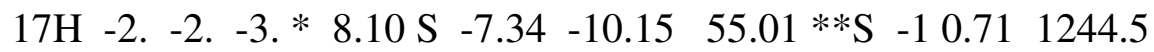
$18 \mathrm{H}-3 . \quad-2 . \quad-2 . * 8.25 \mathrm{~S}-35.75 \quad-12.20 \quad 60.50 * * \mathrm{~S} \quad 20.721817 .7$ $19 \mathrm{H}-1 . \quad 4 . \quad-3 . * 10.04 \mathrm{~S} \quad 20.53 \quad 28.51 \quad-55.15 * * \mathrm{~S} \quad 20.87 \quad 8445.4$ $20 \mathrm{H}-2 . \quad 4 .-2 . * 9.76 \mathrm{~S}-2.02 \quad 27.31-51.90 * * \mathrm{~S} \quad 1 \quad 1.0421079 .8$ $21 \mathrm{H}-3 . \quad 5 . \quad 1 . * 11.90 \mathrm{~S}-43.6929 .79-53.96 * * \mathrm{~S} \quad 11.008956 .4$ $22 \mathrm{H}-3 . \quad 5 . \quad-1 . * 11.90 \mathrm{~S}-20.12 \quad 29.20-52.26 * * \mathrm{~S} \quad 11.158967 .8$ $23 \mathrm{H}-2 . \quad 5 . \quad-3 . * 12.25 \mathrm{~S} \quad 7.61 \quad 30.13-53.93 * * \mathrm{~S} \quad 11.0121325 .2$ $24 \mathrm{H}-2$. $5 . \quad-2 . * 11.47 \mathrm{~S}-0.90 \quad 31.81 \quad-59.61 * * \mathrm{~S} \quad 1 \quad 1.19 \quad 694.0$ $25 \mathrm{H}-1 . \quad 4 . \quad-1 . * 8.45 \mathrm{~S} \quad 0.84 \quad 33.78 \quad-73.19 * * \mathrm{~S} \quad 11.1322465 .5$ $\mathrm{CD} 0>$ reind

$\mathrm{NrS} \quad \mathrm{H} \quad \mathrm{K} \quad \mathrm{L} \quad$ Dev-Ang $\quad \mathrm{dTh} \quad \mathrm{dPh} \quad \mathrm{dCh} 0.0093594$

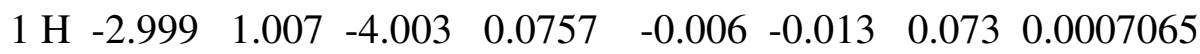
$\begin{array}{lllllllll}2 \mathrm{H} & -1.999 & 1.001 & -5.002 & 0.0211 & -0.002 & -0.012 & 0.017 & 0.0002248\end{array}$ $\begin{array}{lllllllll}3 \mathrm{H} & -4.000 & 0.993 & -3.001 & 0.0819 & 0.003 & -0.020 & -0.078 & 0.0007233\end{array}$ $\begin{array}{lllllllll}4 \mathrm{H} & -4.000 & 1.000 & -4.001 & 0.0109 & -0.002 & -0.011 & -0.008 & 0.0001326\end{array}$ $\begin{array}{lllllllll}5 \mathrm{H} & -0.999 & -0.003 & -5.999 & 0.0329 & 0.003 & -0.018 & -0.029 & 0.0003606\end{array}$ $\begin{array}{llllllllll}6 \mathrm{H} & 0.001 & -0.005 & -5.990 & 0.0476 & 0.019 & -0.020 & -0.042 & 0.0010464\end{array}$ $\begin{array}{lllllllll}7 \mathrm{H} & -2.000 & 0.998 & -6.001 & 0.0226 & -0.001 & -0.008 & -0.0221 & 0.0002412\end{array}$ $\begin{array}{lllllllll}8 \mathrm{H} & -4.002 & 2.009 & -4.002 & 0.0764 & -0.012 & 0.012 & 0.076 & 0.0009725\end{array}$ $\begin{array}{lllllllll}9 \mathrm{H} & -1.000 & 1.001 & -6.002 & 0.0124 & -0.005 & -0.005 & 0.012 & 0.0002521\end{array}$ $\begin{array}{lllllllll}10 \mathrm{H} & -3.998 & -2.004 & -2.001 & 0.0486 & 0.000 & -0.029 & -0.045 & 0.0004099\end{array}$

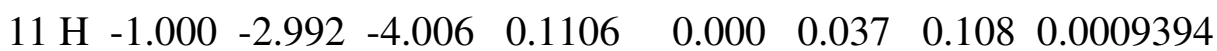
$\begin{array}{lllllllll}12 \mathrm{H} & -4.001 & -2.997 & -3.003 & 0.0351 & -0.001 & -0.011 & 0.033 & 0.0003537\end{array}$

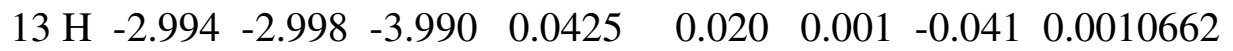
$\begin{array}{lllllllll}14 \mathrm{H} & -0.003 & -3.997 & -5.005 & 0.0578 & -0.002 & 0.063 & 0.033 & 0.0006273\end{array}$ $\begin{array}{lllllllll}15 \mathrm{H} & -4.000 & -2.000 & -3.998 & 0.0161 & 0.003 & 0.014 & -0.012 & 0.0002309\end{array}$ $\begin{array}{lllllllll}16 \mathrm{H} & -2.001 & -2.002 & -3.996 & 0.0523 & 0.004 & 0.020 & -0.055 & 0.0004614\end{array}$

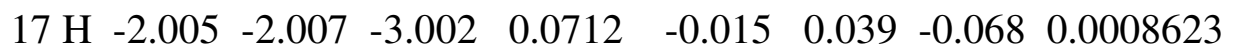
$\begin{array}{lllllllll}18 \mathrm{H} & -3.002 & -2.005 & -2.001 & 0.0525 & -0.009 & -0.002 & -0.053 & 0.0005692\end{array}$ $\begin{array}{lllllllll}19 \mathrm{H} & -1.004 & 3.991 & -3.005 & 0.1136 & 0.007 & -0.024 & -0.112 & 0.0010373\end{array}$ $\begin{array}{lllllllll}20 \mathrm{H} & -1.999 & 4.003 & -2.000 & 0.0272 & -0.005 & -0.002 & 0.028 & 0.0003260\end{array}$ $\begin{array}{lllllllll}21 \mathrm{H} & -3.003 & 4.999 & 1.003 & 0.0405 & -0.002 & 0.034 & -0.032 & 0.0004221\end{array}$ $\begin{array}{lllllllll}22 \mathrm{H} & -3.000 & 5.001 & -0.999 & 0.0141 & 0.000 & 0.016 & 0.008 & 0.0001445\end{array}$ $\begin{array}{lllllllll}23 \mathrm{H} & -1.999 & 5.003 & -3.001 & 0.0257 & -0.005 & -0.010 & 0.024 & 0.0003557\end{array}$ $\begin{array}{lllllllll}24 \mathrm{H} & -1.998 & 5.001 & -1.999 & 0.0216 & 0.000 & 0.003 & 0.021 & 0.0002118\end{array}$

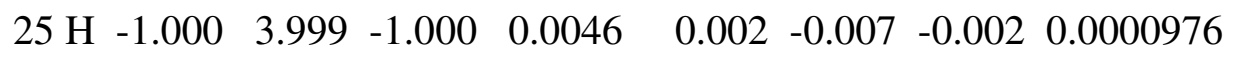
Reciprocal axis matrix Direct axis matrix

$$
\begin{array}{rrrrrr}
-0.076371 & 0.020678 & 0.056979 & -7.659393 & -5.683682 & -3.047902 \\
-0.056719 & 0.022058 & -0.074083 & 2.150182 & 2.314291 & -9.716051 \\
-0.030412 & -0.093093 & -0.005038 & 6.504328 & -8.458021 & -0.559365
\end{array}
$$




\begin{tabular}{cccccc} 
Niggli-values & \multicolumn{5}{c}{ Sigma direct axis matrix } \\
100.2603 & 104.3808 & 114.1574 & 0.002766 & 0.001097 & 0.001836 \\
-0.1541 & -0.0417 & -0.0091 & 0.005859 & 0.002324 & 0.003892 \\
0.004838 & 0.001919 & 0.003213 & & &
\end{tabular}

Cell parameters Sigma cell parameters

\begin{tabular}{llllll}
10.20230 & 10.2067 & 10.5844 & 0.0022 & 0.0038 & 0.0034 \\
90.2008 & 90.0243 & 90.1752 & 0.0286 & 0.0238 & 0.0291 \\
-0.0014141 & -0.000388 & -0.000099 & 0.000499 & 0.000417 & 0.000509 \\
\multicolumn{5}{c}{0.5956} \\
Volume= 1102.1727 \\
Index-Status: HHHHHHHHHHHHHHHHHHHHHHH \\
CD0>
\end{tabular}

The lattice parameters are $a=10.02 \AA, b=10.20 \AA, c=10.58 \AA, \alpha=90.2^{\circ}, \beta=90.0^{\circ}$ and $\gamma=90.1^{\circ}$. The volume of the unit cell of the MnHP crystal is $1102.1727(\AA)^{3}$. From the above data it was confirmed that MnHP crystal system is triclinic [21-22].

\section{CONCLUSION}

The MnHP crystals were grown at room temperature and found optimum growth parameters. MnHP crystal growth columns and harvested crystal are photographed. FTIR-spectrum recorded the functional group frequencies of MnHP grown crystal constituents. These results were recorded and compared with the reported values. Chemical etching was done at room temperature, which revealed the grown crystal defects. SEM analysis was also done and it revealed the surface morphology of MnHP crystal. MnHP lattice parameters were calculated by XRD.

\section{REFERENCE}

[1] Sundaramoorthi P., and Kalainathan S., 2007, Biochemical Engineering journal, Vol. 34, pp. 244.

[2] Sundaramoorthi P., and Kalainathan S., 2007, Journal of Materials and Minerals and Characterization of Engineering, Vol. 6, pp. 33.

[3] Sundaramoorthi, P., and Kalainathan S., 2007, Asian journal of chemistry, Vol. 19, pp. 3739.

[4] Henisch H. K., Garcia-Ruiz J.M., 1986, J. Crystal Growth, Vol. 75, pp. 195.

[5] Henisch H.K., Garcia-Ruiz J.M., 1986, J. Crystal Growth, Vol. 75, pp. 203.

[6] Pecsok R.L., Shields L.D., Cairns T., and McWillian I.G., 1976, Modern methods of chemical analysis, John Wily\&Sons Inc.,Newyork, pp. 438-442. 
[7] Henisch H.K., Crystals in Gel and Liesegang Rings, 1986, Cambridge University press, Cambridge, pp. 11.

[8] Socrater, G., 1980, Infrared Cha. Group Friq., Johnwilly- Chichester.

[9] Hatscheck E., 1925, J. Kollid Z., Vol. 37, pp. 297.

[10] Chin Y., et al., 1961, J. Urology, Vol. 86, pp. 838-854.

[11] Corns C.M., 1983, J. Ann. Clin. Bio-Cherm,, Vol. 20, pp. 20-35.

[12] Hesse A., and Bach D., 1982, Stone analysis by infrared spectroscopy, In., Urinary stones, Clinical and laboratory Aspects, Edi., Alan Rose, University Park Press, Baltimore, Vol. 87, pp. 105.

[13] Gilman, J.J., Johnsion J., and Sears G.W., 1958, J. App. Physics, Vol. 29, pp. 749.

[14] Gilman J.J., et al, 1956, J. App. Physics, Vol. 27, pp. 1018.

[15] Fisher J.C., 1957, Dissolutions and Mechanical Properties of Crystals, John Wiley and sons, New York.

[16] Kirk J.B., 1962, Director Observation of Imperfection in Crystals, Inter science Publishers, New York.

[17] Taukamot K., 1983, J. Crystal. Growth, Vol. 61, pp. 199.

[18] Gates H.C., 1975, Thirty years of progress in Surface Science, Crystal growth and Characterization, Edi., North Holland.

[19] Bethage H., et al., 1987, Electron Microscopy in Solid State Physics, Elsevier, Amsterdom.

[20] Albon N. et al, 1958, Growth and Perfection of Crystals, Wiley., New York.

[21] Machennan G., and Reevers C.A., 1955, Acta Crystals, Vol. 8, pp. 579.

[22] Curry N.A., and Jones D.W., 1971, J. Chem. Soc. A, pp. 3725. 